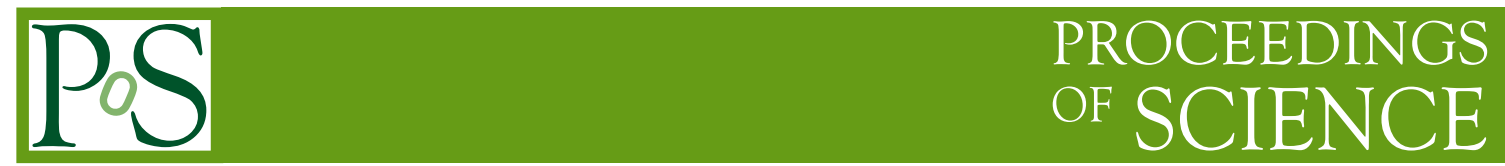

\title{
Spectral triples and Toeplitz operators
}

\author{
Kevin Falk* \\ Centre de Physique Théorique, Marseille, France \\ E-mail: kevin.falkecpt.univ-mrs.fr
}

\begin{abstract}
We present the main results of [6] on the construction of spectral triples using the algebra generated by Toeplitz operators acting on Fock spaces and Hardy and Bergman spaces over a strictly pseudoconvex domain of $\mathbb{C}^{n}$. Different Dirac operators are proposed and we compare their influence on the dimension of the corresponding spectral triple. Since Toeplitz operators play an important role in deformation quantization, we also study how Berezin-Toeplitz quantization can be incorporated in the framework of noncommutative geometry.
\end{abstract}

Frontiers of Fundamental Physics 14

15-18 July 2014

Aix Marseille University (AMU) Saint-Charles Campus, Marseille, France

\footnotetext{
* Speaker.
} 


\section{A quick overview of noncommutative geometry}

The field of noncommutative geometry (NCG) gives a mathematical framework to describe geometric entities with algebraic tools. The starting result of NCG is the Gelfand-Naimark theorem [8], which stays a commutative $\mathrm{C}^{*}$-algebra, unital or not, is isometrically *-isomorphic to $C_{0}(X)$, the set of continuous functions vanishing at infinity on some locally compact Hausdorff space $\mathrm{X}$. Conversely, given $\mathrm{X}$, then $C_{0}(X)$ is trivially commutative. Roughly speaking, it means that geometric spaces can be entirely characterized as a commutative algebras.

Connes' idea [4] was to consider not commutative algebras anymore but rather noncommutative ones, and study the corresponding "noncommutative space". From a physicist's standpoint, NCG has natural applications in quantum mechanics, in which observables do not commute.

The main geometric information are encoded in the principal object of NCG, a spectral triple $(\mathscr{A}, \mathscr{H}, \mathscr{D})$, consisting in

- an involutive algebra $\mathscr{A}$,

- a faithful representation $\pi$ of $\mathscr{A}$ by bounded operators on a Hilbert space $\mathscr{H}$,

- a selfadjoint operator $\mathscr{D}$ acting on $\mathscr{H}$ such that for any $a \in \mathscr{A}$, the extended operator of $[\mathscr{D}, \pi(a)]$ is bounded, and $\pi(a)\left(1+\mathscr{D}^{2}\right)^{-1 / 2}$ is compact.

Also, the notion of dimension is given by the quantity $\inf \left\{s \in \mathbb{R}, \operatorname{Tr}\left(|\mathscr{D}|^{-s}\right)<+\infty\right\}$.

We are interested in our case in algebras related to the so-called Toeplitz operators acting on Hilbert spaces of holomorphic functions over strictly pseudoconvex manifolds in $\mathbb{C}^{n}$ or $\mathbb{C}$ itself.

\section{Deformation quantization and Toeplitz operators}

Geometric quantization [5] describes relations between classical and quantum mechanics by studying geometric aspects of a dynamical system. In this context, symplectic manifolds play the role of phase spaces of the system and holomorphic sections represent classic observables. Later on, deformation quantization (DQ) [1] emerged to avoid some technical drawbacks of the geometric quantization. Mimicking the Weyl quantization [7] on flat phase spaces, DQ constructs a formal associative noncommutative star product $\star_{h}$ on the Poisson algebra $\left(C^{\infty}(\Omega),\{.,\}.\right)$ of classical observables over some symplectic phase space $\Omega$, such that, in a suitable sense

$$
f \star_{h} g=\sum_{j \in \mathbb{N}} h^{j} C_{j}(f, g), \quad \text { as } h \rightarrow 0,
$$

where the bilinear operators $C_{j}$ verify

$$
C_{0}(f, g)=f g, \quad C_{1}(f, g)-C_{1}(g, f)=-\frac{i}{2 \pi}\{f, g\}, \quad C_{j}(f, 1)=C_{j}(1, f)=0 \forall j \in \mathbb{N} .
$$

In this context, Toeplitz operators allow to construct the so-called Berezin-Toeplitz star product [3] for phase spaces modeled by pseudoconvex domains.

We consider an open smoothly bounded strictly pseudoconvex manifold $\Omega \subset \mathbb{C}^{n}$ and fix a defining function $r$, i.e. verifying $\left.r\right|_{\Omega}>0,\left.r\right|_{\partial \Omega}=0$ and $\left.\partial r\right|_{\partial \Omega} \neq 0$. Three Hilbert spaces will be used:

- Hardy space $H^{2}$ : the set of boundary values of holomorphic functions on $\Omega$. 
- Bergman space $A_{m}^{2}$ : holomorphic functions in $L^{2}(\Omega, w(z) d z)$, with a weight of the form $w=r^{m} g, 0<g \in C^{\infty}(\Omega), m>-1$.

- Fock space $F_{\rho}$ : holomorphic functions in $L^{2}\left(\mathbb{C}^{n}, \rho(z) e^{-|z|^{2}} d z\right)$, with $0<\rho \in C^{\infty}\left(\mathbb{C}^{n}\right)$.

An interesting property we will use later on is that there are unitaries between $L^{2}\left(\mathbb{R}^{n}\right)$ and all these Hilbert spaces (moreover we have explicit formulas when $\Omega=\mathbb{B}^{n}$ is the unit ball of $\mathbb{C}^{n}$ ).

A usual Toeplitz operator on $H^{2}$ is of the form $T_{u}: v \in H^{2} \mapsto \Pi(u v) \in H^{2}$, with $u \in C^{\infty}(\partial \Omega)$ (Toeplitz on the Bergman and Fock spaces are defined similarly). Toeplitz have properties $\left(u \mapsto T_{u}\right.$ is linear, $T_{u}^{*}=T_{\bar{u}},\left\|T_{u}\right\| \leq\|u\|_{\infty}$, etc.) that are interesting in both NGC and DQ, but one of the main difficulty is that they do not form an algebra since in general $T_{u} T_{v} \neq T_{w}$.

Boutet de Monvel and Guillemin extended Toeplitz operators to Generalized Toeplitz operators (GTOs) [2], that is, operators of the form $T_{Q}: u \in H^{2} \mapsto \Pi(Q u) \in H^{2}$, where $Q$ is a pseudodifferential operator (YDO) on $\partial \Omega$. The GTOs enjoy useful properties which allow us to build spectral triples. The following table gives the principal ones

\section{Properties}

The set of all GTOs form a filtered algebra

Locally, $G T O(\partial \Omega) \approx \Psi \mathrm{DO}\left(\mathbb{R}^{\mathrm{n}}\right) \bmod$ smoothings

$\forall T_{Q}, \exists P$ s.t. $\operatorname{ord}(Q)=\operatorname{ord}(P), T_{Q}=T_{P}$ and $[\Pi, P]=0$

Existence of a principal symbol: $\sigma\left(T_{Q}\right):=\left.\sigma(Q)\right|_{\Sigma}{ }^{(*)}$

Dixmier trace: $\operatorname{Tr}_{\omega} T_{Q}=\frac{1}{n !(2 \pi)^{n}} \int_{\partial \Omega} \sigma\left(T_{Q}\right)\left(x, \eta_{x}\right)$

\section{Interests for NCG}

Good candidate for $\mathscr{A}$

Links with known spectral triples

Control the axioms of a spectral triple

Allows a symbolic calculus

Computation of the dimension

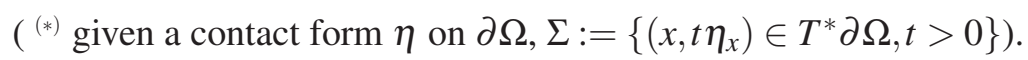

\section{Examples of spectral triples}

Let us describe now different spectral triples involving algebras generated by the Toeplitz operators of different kinds. The principal example of spectral triple over $\partial \Omega$ is the following:

- $\mathscr{A}$ : the set of GTOs of order $\leq 0$,

- $\mathscr{H}=H^{2}(\partial \Omega)$

- $\mathscr{D}$ : an elliptic selfadjoint GTO of order 1 ,

form a spectral triple of dimension $n=\operatorname{dim}_{\mathbb{C}} \Omega$. The main steps to prove this are the following: $\mathscr{A}$ is trivially an involutive algebra with representation the identity on $\mathscr{H}, \mathscr{D}$ is elliptic with $\mathscr{D}^{-1} \in$ $G T O^{-1}$ hence has compact resolvent, for any $T_{P} \in \mathscr{A},\left[\mathscr{D}, T_{P}\right] \in G T O^{0}$ so is bounded, and finally the Weyl formula for GTOs leads us to the dimension.

To take an example of Dirac operator in the case $\Omega=\mathbb{B}^{n}$, we can use the usual Dirac on $\mathbb{R}^{n}$ $\left(\not D=\gamma^{\mu} \partial_{\mu}\right)$ transported on $H^{2}$ via the known unitaries. We then obtain a spectral triple of dimension $2 n=\operatorname{dim}_{\mathbb{R}} \Omega$. This difference in the dimension comes from the fact that a $\Psi$ DO of order $k$ on $\mathbb{R}^{n}$ is transformed to a GTO of order $k / 2$.

The next spectral triple concerns the domain $\Omega$ itself:

- $\mathscr{A}$ : the algebra generated by Toeplitz operators $T_{f}, f \in C^{\infty}(\bar{\Omega})$,

- $\mathscr{H}$ : the weighted Bergman space $A_{m}^{2}$, 
- $\mathscr{D}=V T V^{-1}$, where $T$ is a selfadjoint elliptic GTO of order 1 , and $V: A_{m}^{2} \rightarrow H^{2}$ is a unitary, form a spectral triple of dimension $n$. The key point of the proof is to express the objects in terms of GTOs on $H^{2}$ via the unitaries. As before, in the case $\Omega=\mathbb{B}^{n}, w=1$, the usual Dirac $\not D$ to the Bergman space via unitaries induces a spectral triple of dimension $2 n$.

Another example of (positive) Dirac is simply $\mathscr{D}=T_{r}^{-1}$. Some extensions with non positive $\mathscr{D}$ are possible by adding some unitary operators to the Dirac and by Hilbert doubling.

On the Fock space on $\mathbb{C}$, we have the spectral triple of dimension $2 \operatorname{dim}_{\mathbb{R}} \mathbb{C}$, formed by

- $\mathscr{A}=\left\{T_{f}, f \in \cup_{s \leq 0} E^{s}\right\}, E^{s}$ be the set of functions on $\mathbb{C}$ verifying $f(z) \sim|z|^{s} \sum_{k=0}^{\infty} f_{k}\left(\frac{z}{|z|}\right)|z|^{-k}$ as $|z| \rightarrow \infty$, with $s \in \mathbb{R}$ and $f_{k}$ smooth on $S^{1}$,

- $\mathscr{H}=F_{\rho}$,

- $\mathscr{D}=T_{g}, g \in E^{1}$ and measurable,

The proof uses some relations between Toeplitz on Fock spaces and Weyl operators on $\mathbb{R}^{2}$ (which enjoys properties similar to $\Psi D O s)$.

Finally, let us describe a spectral triple obtained from the Berezin-Toeplitz quantization. It can be shown that GTOs can also be defined on the Hardy space $\widetilde{H}^{2}$ over $\partial \widetilde{\Omega}$, where $\widetilde{\Omega}$ is a disk bundle of $\Omega$. It happens that $\Lambda=K^{*} K$ is an elliptic selfadjoint pseudodifferential operator on $L^{2}(\partial \widetilde{\Omega})$ of order -1 , where $K$ is the Poisson extension operator. From the fact that the spaces $\widetilde{H}^{2}$ and $\bigoplus_{m \in \mathbb{N}} A_{m}^{2}(\Omega)$ are unitarily equivalent, we get the following result:

- $\mathscr{A}$ : the algebra generated by the Toeplitz of the form $T_{f}^{\oplus}=\bigoplus_{m \in \mathbb{N}}\left(T_{f}\right.$ on $\left.A_{m}^{2}(\Omega)\right)$,

- $\mathscr{H}=\bigoplus_{m \in \mathbb{N}} A_{m}^{2}(\Omega)$,

- $\mathscr{D}$, the operator on $\mathscr{H}$ corresponding to $T_{\Lambda}^{-1}$ via the mentioned unitary,

form a spectral triple of dimension $n+1$. To prove this, we use the result of Berezin-Toeplitz quantization about the existence of a start product $\star$ such that $T_{f}^{\oplus} T_{g}^{\oplus} \underset{m \rightarrow \infty}{\sim} T_{f \star g}^{\oplus}$.

\section{References}

[1] F. Bayen, M. Flato, C. Fronsdal, A. Lichnerowicz, D. Sternheimer, Deformation theory and quantization, Ann. Phys. 111 (2), 6-110 (part I), 111-151 (part II), 1978

[2] L. Boutet de Monvel and V. Guillemin, The spectral theory of Toeplitz operators, Princeton University Press, Princeton, N.J., 1981.

[3] M. Bordemann, E. Meinrenken and M. Schlichenmaier, Toeplitz quantization of Kähler manifolds and $g l(N), N \rightarrow \infty$ limits, Comm. Math. Phys., 165, 281-296, 1994.

[4] A. Connes, Noncommutative geometry, Academic Press, 1994.

[5] A. Echeverría-Enriquez, M. C. Muñoz-Lecanda, N. Román-Roy, C. Victoria-Monge, Mathematical foundations of geometric quantization, Extracta Mathematicae, 13, 235-238, 1998.

[6] M. Engliš, K. Falk, B. Iochum, Spectral triples and Toeplitz operators, arXiv:1402.3061.

[7] H. J. Groenewold, On the Principles of Elementary Quantum Mechanics, Physica, 12, 405-460, 1946.

[8] I. M. Gelfand, M. A. Naimark, On the imbedding of normed rings into the ring of operators on a Hilbert space, Math. Sbornik, 12 (2), 197-217, 1943. 